\title{
A New Efficient Agent to Chemo-mechanical Caries Removal
}

\author{
Sandra Meyfarth, ${ }^{1}$ Karen Cassano, ${ }^{2}$ Flavio Warol, ${ }^{3}$ Marcia de Deus Santos, ${ }^{3}$ Angela Scarparo ${ }^{2,4}$ \\ ${ }^{1}$ Postgraduation Program, School of Dentistry, Fluminense Federal University, Niterói, RJ, Brazil \\ ${ }^{2}$ School of Dentistry, Fluminense Federal University, Nova Friburgo, RJ, Brazil \\ ${ }^{3}$ Postgraduation Program, School of Dentistry, Grande Rio University, Duque de Caxias, RJ, Brazil \\ ${ }^{4}$ Postgraduate Program in Dentistry of Health Institute of Nova Friburgo (PPGO-ISNF), Fluminense Federal University, Nova Friburgo, RJ, Brazil \\ - Conflicts of interest: none declared.
}

\section{Abstract}

Objective: The aim of this study was to perform a brief literature review of chemo-mechanical caries removals and to describe a clinical case using the product. Case report: According to clinical and radiographic diagnosis, the tooth presented dentin caries lesion without any pulpal involvement. BRIX $3000^{\circledR}$, a new chemo-mechanical caries removal product, was used with 1 gel application of 2 minutes. Afterwards, the cavity was restored using a bulk fill flow composite and a conventional resin composite final layering. Conclusion: Taking into consideration the presented clinical case, the application of BRIX $3000^{\circledR}$ gel seems to be a good alternative for caries removal followed by a restorative treatment, since the child's discomfort decreased, resulting in a positive behavior. BRIX 3000 is an efficient chemo-mechanical caries removal method. Keywords: Chemo-mechanical caries removal; Brix 3000; Deciduous tooth; Dental caries; Pediatric dentistry.

\section{Introduction}

1 The development of techniques to remove caries is advancing towards a more conservative and biological direction. ${ }^{1,2}$ Conventional restorative procedures suggest the complete elimination of the cariogenic biomass, also involving the removal of excessive sound tissues. With the advance of the minimally invasive dentistry, some studies indicate that the removal of all contaminated tissue is not essential to stop the cariogenic process. ${ }^{3,4}$ The partial removal of the carious tissue aims to maintain the pulp integrity. It is considered as the therapy of choice for treating acute and deep lesions, since some principles of diagnosis are respected. ${ }^{5,6} \mathrm{The}$ carious dentine is completely removed from the surrounding cavity walls followed by the more necrotic and infected dentin at the pulpal wall. Afterwards, the tooth restoration is performed. ${ }^{6}$

Finding effective and less invasive methods of caries removal have been a major objective for dental researchers. ${ }^{1,2}$ Caries removal using the conventional method is an unpleasant experience. The pain generated by drilling due to thermal changes and vibrations during cavity cutting may cause discomfort to the patient ${ }^{7}$ and have led to the introduction of the chemo-mechanical approach as a noninvasive alternative for the removal of carious dentin., ${ }^{78}$ Chemo-mechanical caries removal provokes the chemical softening of carious dentin to be subsequently removed by a gentle manual excavation. It involves the selective removal of degraded collagen fibrils in carious dentin lesion, while preserving the affected demineralized dentin layer. ${ }^{9}$
A clinically effective caries removal reagent harmless to healthy tissue and biocompatible to the pulp is fundamentally difficult to develop. ${ }^{1}$ Since the 1970, chemical compositions have been used for chemo-mechanical caries removal. ${ }^{9}$ In 1975, the first chemo-mechanical caries removal method by using sodium hypochlorite was patented. ${ }^{10}$ In 1985 , Caridex $^{\mathrm{TM}}$ system was introduced in the market, resulting of studies to improve the efficacy of sodium hypochlorite solution. Nevertheless, the product had some disadvantages, e.g. short expiry date, large volume of solution required and preheating. ${ }^{11}$ Then, in 1998 a new material, Carisolv ${ }^{\mathrm{TM}}$ system, (MediTeam, Goteborg, Sweden) hit the market. ${ }^{12}$ It is the only hypochlorite based chemo-mechanical caries removal commercially available. ${ }^{13}$ Despite the effectiveness of Carisolv ${ }^{\mathrm{TM}}$, it has some limitations such as the high cost, certification of dental surgeons and use of special instruments. ${ }^{12}$ The first enzyme-based commercially available chemo-mechanical caries removal agent is Papacarie ${ }^{\circledR}$, introduced in 2003. It is basically comprised of papain, chloramines, toluidine blue, salts, thickening vehicle, giving bactericide, bacteriostatic and anti-inflammatory properties. ${ }^{11}$ Papacarie ${ }^{\circledast}$ has some advantages such as easy application, waiting time after application of 30 to 60 seconds, no need for special equipment to apply it no need of local anesthesia and use of drill. ${ }^{5}$

BRIX $3000^{\circledR}$ (BRIX SRL and BRIX USA LLC), a new chemo-mechanical caries removal launched in Argentina in 2016, is a gel product also derived from fruits of green papaya. It involves an enzymatic activity $\left(3.000 \mathrm{U} / \mathrm{mg}^{*}\right)$ in which 
the papain is bio-encapsulated by using EBE Technology (Encapsulating Buffer Emulsion) that immobilizes and confers stability, increasing the enzymatic activity of the final product exponentially. According to the manufacturer, it is easily applied, with no need of special instruments and waiting time after application of 2 minutes.

Few studies evaluated the effectiveness of chemomechanical caries removal. ${ }^{14,15}$ These studies observed an effective removal of the carious dentine in primary teeth without affecting negatively the children's cooperation. ${ }^{14}$

The aim of this study was to perform a brief literature review of the chemo-mechanical caries removals with a case report using a new chemo-mechanical agent, BRIX $3000^{\circledR}$.

\section{Case report}

A seven years old boy was referred to the Pediatric Clinic at a Public University. Clinically, the permanent left lower first molar (36) presented an acute caries lesion with easy access (Fig. 1A). After a detailed explanation of the treatment plan, a consent form was signed authorizing the attendance and photographic documentation of the case. Only the gingival papillae region was anesthetized with $2 \%$ Lidocaine, to become possible the placement of the clamp number W84 (SS White Duflex, Brazil) and the rubber dam (Madeitex, Brazil) (Fig. 1C). Then, the tooth prophylaxis was performed followed by the application of the caries removal gel, BRIX $3000^{\circledR}$, according to the manufacturer's instructions
(Fig. 1D). The gel remained into the cavity for two minutes. Soon after, the removal of carious dentin was performed carefully with hand excavator. The consistency of the carious tissue was soft and easily removed. There was no need to reapply the product since it was possible to remove the entire carious lesion in a single application. Figure $1 \mathrm{E}$ shows the complete removal of the carious lesion. The tooth restoration was performed starting with the selective enamel conditioning with $37 \%$ phosphoric acid (Dentsply, Brazil) (Fig. 1F). After 15 seconds the gel was washed away with water for 30 seconds and then the tooth was dried carefully until the total evaporation of water from the cavity surface. The self-etching adhesive system Ambar Universal (FGM, Brazil) was actively applied over the cavity surface for 20 seconds, according to the manufacturer's instructions. The disposable brush was agitated on the cavity surface under manual pressure. Then, for complete evaporation of the solvent, a moderate jet of clean, dry air was applied. The adhesive was photo activated for 10 seconds and the tooth was restored. The bulk fill flow resin composite (Opus Bulk Fill Flow - FGM, Brazil) was used in a single increment (Fig. 1G) finishing with a conventional resin composite layer (Opallis - FGM, Brazil) (Figs. 1H-1I).

\section{Discussion}

Rotary instruments associated with problems such as pulp overheating, need for local anesthesia and discomfort

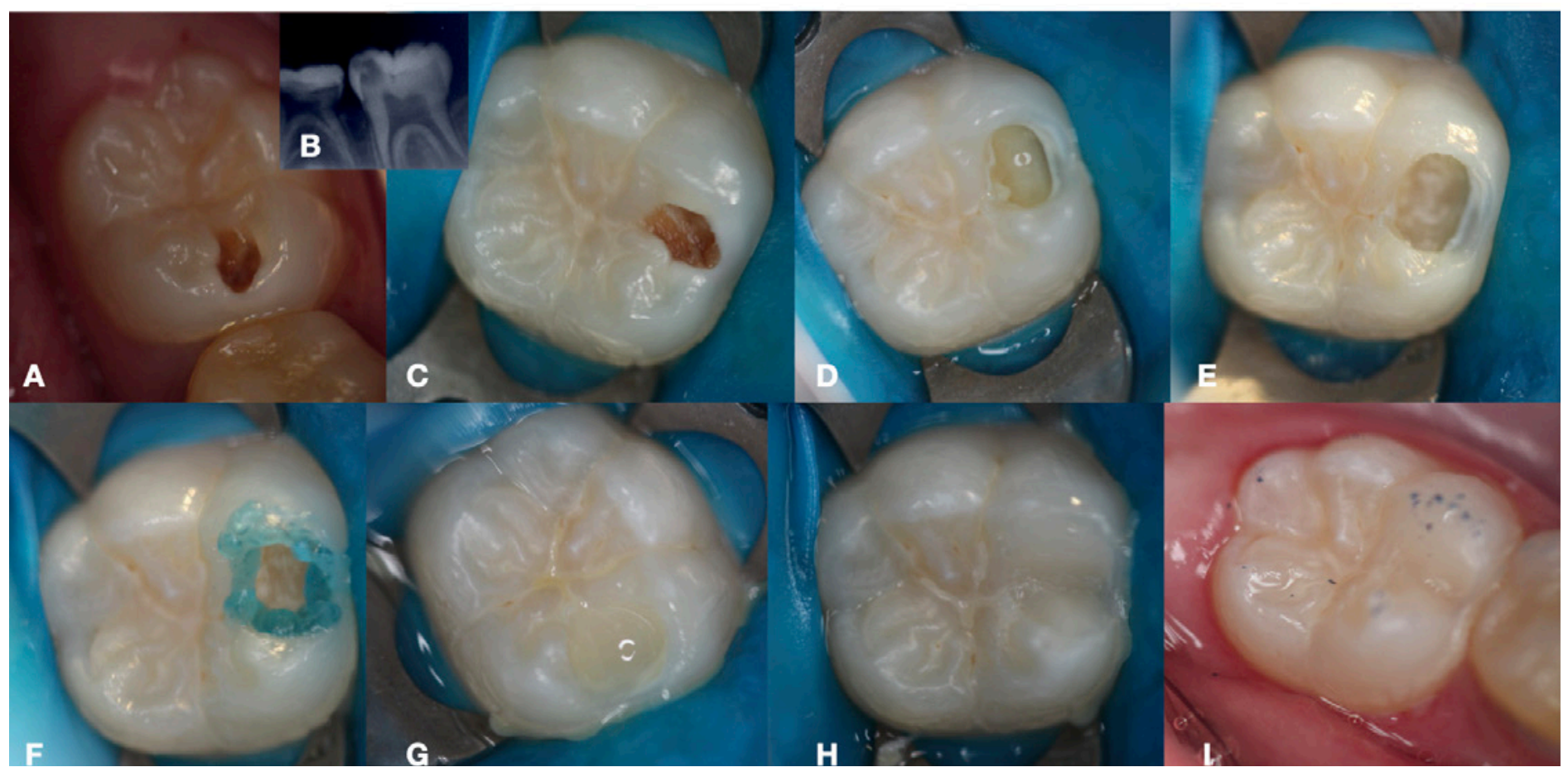

Figure 1. Clinical case illustrative figures: 1A: Initial aspect of caries lesion; 1B: Radiographic image showed a medium caries lesion, with easy access and absence of pulp involvement; 1C: Rubber dam of dental element; 1D: Application of the caries removal gel, BRIX 3000 ${ }^{\circledR}$, according to the manufacturer's instructions; $1 \mathrm{E}$ : Final aspect after complete removal of the carious lesion; $1 \mathrm{~F}$ : Selective enamel conditioning with $37 \%$ phosphoric acid, for 15 seconds; 1G: Bulk fill flow resin composite in a single increment; $1 \mathrm{H}$ : Finishing with a conventional resin composite layer; 1l: Final aspect of restorative procedure. 
lead to increase the patient's anxiety during treatment. ${ }^{16}$ Due to the disadvantages of conventional techniques, especially unnecessary removal of sound tissues, restorative dentistry has focusing on teeth preservation and minimally invasive techniques, searching new methods for the removal of

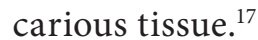

Chemo-mechanical caries removal is a conservative and convenient caries removal method. ${ }^{8}$ It was introduced nearly three decades ago and is a non-invasive alternative for the removal of carious dentine. The method involves to apply a solution onto the decayed dentin allowing it to soften, and, then, to scrap it off with hand excavators. The partially degraded collagen in carious dentine is chlorinated which affects the secondary and/or quaternary structure of collagen, by disrupting hydrogen bonding, allowing an easier removal of carious tissue. ${ }^{18}$ This method has some benefits including no damage to sound tooth structure, reduced pulpal irritation and pain compared to conventional methods and an absence of smear layer. The chemo-mechanical caries removal technique may be a useful adjunct treatment, especially to non-cooperative patients who present dental fear and anxiety and phobia of needles.

The new chemo-mechanical caries removal, BRIX $3000^{\circledR}$, was an efficient method to remove dentin caries. The explorer passed smoothly over the remaining dentin surface, as the carious lesion was open and accessible for hand instrument. Also, its consistency was either soft or medium. The complete caries removal was assessed visual and tactile and no drill was used. This is very positive, once drills remove not only infected dentin, but also affected dentin simultaneously, with possible extension into the underlying sound dentin. This is usually accompanied by pain and need of local anesthesia application ${ }^{1}$. In the case reported, there was absence of pain during the procedure and no need of extra anesthesia. This might be due to the fact that Papain softens only the infected layer of carious dentin, which has no odontoblastic processes being insensitive. ${ }^{19}$ According to the manufacturer, papain is the main component of BRIX $3000^{\circledR}$, and it is bio encapsulated with the E.B.E. technology (Encapsulating Buffer Emulsion), which immobilizes the gel and confers stability increasing the enzymatic activity of the product exponentially. Papain is an enzyme, similar to human pepsin, and it breaks denatured collagen fibers acting upon the contaminated dentin, allowing easy removal with hand pieces. ${ }^{20}$

Additionally, in this case, the operative time to remove the caries lesion did not take more than five minutes, which contributed for the child's behavior, confirming findings. ${ }^{15}$

\section{Conclusion}

This study indicated that BRIX $3000^{\circledR}$ is an efficient caries removal. The new chemo-mechanical agent appears to be comfortable, with no need of anesthesia to execute the procedure. The technique is simple and do not need any training. It seems to be an alternative and effective method to treat caries lesions in children, especially those with behavior problems. However, further studies should be carried out in order to enable the use of this new product in the clinical routine.

\section{References}

1. Kotb RMS, Abdella AAH, El Kateb M A, Ahmed AM. Clinical Evaluation of Papacarie in Primary Teeth. J Clin Pediatr Dent 2009; 34(2):117-24.

2. Bjorndal L, Simon S, Tomson PL, Duncan HF. Management of deep caries and the exposed pulp. Int Endo J 2019;52(7):949-73.

3. Maltz M, Oliveira EF, Fontanella V, Carminatti G. Deep Caries Lesions after Incomplete Dentine Caries Removal: 40-Month Follow-Up Study. Caries Res 2007;41:493-6.

4. Santos PS, Pedrotti D, Braga MM, Rocha RO, Lenzi TL. Materials used for indirect pulp treatment in primary teeth: a mixed treatment comparisons meta-analysis. Braz Oral Res 2017 Dec;31:e101.

5. Bottega F, Bussadori SK, Battisti IDE, Vieira EP, Pompeo TS, Winkelmann ER. Costs and benefits of Papacarie in pediatric dentistry: a randomized clinical trial. Sci Rep 2018;17;8(1):17908.

6. Maltz M, Koppe B, Jardim JJ et al. Partial caries removal in deep caries lesions: a 5-year multicenter randomized controlled trial. Clin Oral Invest 2018;22(3):1337-43.

7. Venkataraghavan K, Kush A, Lakshminarayana C, et al. Chemomechanical caries removal: a review \& study of an indigenously developed agent (Carie Care $^{\mathrm{Tm}}$ in children. J Int Oral Health 2013;5(4):84-90.

8. Hegde S, Kakti A, Bolar DR, Bhaskar SA. Clinical Efficiency of Three Caries Removal Systems: Rotary Excavation, Carisolv, and Papacarie. J Dent Child 2016;83(1):22-8.

9. Beeley JA, Yip HK, Stevenson AG. Chemochemical caries removal: a review of the techniques and latest developments. Br Dent J 2000;188(8):427-30

10. Habib CM, Kronman J, Goldman M. A chemical evaluation of collagen a hydroxyproline after treatment with GK-101 (N-Chloroglycine). Pharmacol Ther Dent 1975;2:209-15.
11. Bussadori SK, Castro LC, Galvão AC. Papain Gel: A New ChemoMechanical Caries Removal Agent. J Clin Pediatr Dent 2005; 30(20):115-20. 12. Carrilo CM, Tanaka MH, Cesar MF, et al. Use of papain gel in disabled patients. J Dent Child 2008;75(3):222-8.

13. Hamama H, Yiu C, Burrow M. Current update of chemomechanical caries removal methods. Aust Dent J 2014;59(4):446-56.

14. Alkhouli MM, Al Nesser SF, Bshara NG, AlMidani AN, Comisi JC. Comparing the efficacies of two chemo-mechanical caries removal agents (2.25\% sodium hypochlorite gel and brix 3000$)$, in caries removal and patient cooperation: A randomized controlled clinical trial. J Dent 2020;93:103280.

15. Santos TML, Bresciani E, Matos FS et al. Comparison between conventional and chemomechanical approaches for the removal of carious dentin: an in vitro study. Sci Rep 2020;10(1):8127.

16. AlHumaid J, Al-Harbi F, El Tantawi M, Elembaby A.X-ray microtomography assessment of Carisolv and Papacarie effect on dentin mineral density and amount of removed tissue. Acta Odontol Scand 2018;76(4):236-40.

17. Avinash A, Grover SD, Koul M, et al. Comparison of mechanical and chemomechanical methods of caries removal in deciduous and permanent teeth: a SEM study. J Indian Soc Pedod Prev Dent 2012;30(2):115-21.

18. Maragakis GM, Hahn P, Hellwig E. Chemomechanical caries removal: A comprehensive review of the literature. Inter J Dent 2001; 51(4):291-9.

19. Yamada T, Nakamura K, Iwaku M, Fusayama T. The extent of the odontoblastic process in normal and carious dentin. J Dent Res 1983;62:798802 .

20. Bussadori SK, Guedes CC, Bachiega JC, Santis TO, Motta LJ. Clinical and radiographic study of chemical-mechanical removal of caries using Papacarie: 24-month follow up.J Clin Pediatr Dent 2011;35(3):251-4. 


\section{Mini Curriculum and Author's Contribution}

1. Sandra Meyfarth - DDS; MSc. Contribution: Technical procedures, preparation and draft of the manuscript, critical review and final approval. ORCID: 00000003-0074-642X

2. Karen Cassano - DDS. Contribution: Technical procedures, preparation and draft of the manuscript. ORCID 0000-0002-3376-327X

3. Flavio Warol - DDS; MSc. Contribution: Critical review and final approval. ORCID: 0000-0002-2662-751X

4. Márcia de Deus Santos - Preparation and draft of the manuscript, ORCID 0000-0003-3789-4346

5. Angela Scarparo - DDS; PhD. Contribution: Technical procedures supervision; preparation and draft of the manuscript; critical review and final approval. ORCID: 0000-0002-4415-1893

Submitted: 10/17/2020 / Accepted for publication: 12/08/2020

Corresponding author:

Angela Scarparo

E-mail: angelascarparo@id.uff.br 